\title{
REVIEW
}

\section{Laparostomy: why and when?}

\author{
Ari K Leppäniemi* \\ This article is one of ten reviews selected from the Yearbook of Intensive Care and Emergency Medicine 2010 (Springer Verlag) and co-published \\ as a series in Critical Care. Other articles in the series can be found online at http://ccforum/series/yearbook. Further information about the \\ Yearbook of Intensive Care and Emergency Medicine is available from http://www.springer.com/series/2855.
}

\section{Introduction}

Laparostomy is a surgical treatment method in which the peritoneal cavity is opened anteriorly and deliberately left open, hence often called 'open abdomen'. The abdominal contents are exposed and protected with a temporary coverage. The term does not include full-thickness abdominal wall defects resulting from partial excision due to tumor or necrotizing infection, or incisional hernias.

Laparostomy is currently used in many severely ill or injured patients to facilitate healing or prevent complications, most notably the development of abdominal compartment syndrome. It is, however, a morbid procedure with postoperative care that requires good knowledge and skills to prevent even more severe complications. It is also resource intensive, often requiring multiple visits to the operating room and extensive nursing care. With improved understanding of the pathophysiology of common abdominal emergencies, such as abdominal sepsis, severe acute pancreatitis, and major abdominal trauma, as well as their relation to abdominal compartment syndrome, the number of patients with laparostomy can be expected to increase in general and surgical intensive care units.

\section{Who started laparostomy?}

In modern times, the idea of leaving the abdomen open dates back to the 1970s when patients with septic abdomens were treated with laparostomy, in analogy to incision and drainage of an abscess. Similarly to draining an abscess with a large incision and leaving it to heal by secondary intention, open management with frequent dressing changes to clear the infection was used in patients with peritonitis or pancreatitis [1-3].

*Correspondence: ari.leppaniemi@hus.fi

Department of Surgery, Meilahti Hospital, University of Helsinki, Haartmaninkatu 4, PO Box 340, 00029 Hus, Finland
Although the concept of packing the liver after severe trauma was already described in the early 1900s by Pringle and Halsted, the current practice was defined in the 1990s with the concept of damage control surgery, a staged approach to abdominal trauma patients with severe physiological derangement [4]. An important part of the initial, life-saving operation to control bleeding and contamination is to leave the abdomen open for planned relaparotomy 1-2 days later.

Finally, with the recognition of the risks of intraabdominal hypertension (IAH), and the full-blown abdominal compartment syndrome, opening the abdomen and leaving it open has multiplied the numbers of patients with laparostomies [5].

\section{Temporary abdominal cover}

After the initial decision to open the abdomen and/or to leave it open, the exposed viscera must be covered with a protective dressing of some sort to prevent drying and unintentional injury, and to prevent or reduce the risk of infection. Ideally, this dressing should be easy to apply and remove, allow easy nursing care, not damage the fascia or the skin, be readily available and inexpensive, and maintain the abdominal domain. Furthermore, providing easy access to the abdominal cavity and a high rate of subsequent closure of the abdomen, especially the fascia, are additional points to consider.

Excluding the application of a simple dressing used in the early days, the first and easiest method to cover and protect the laparostomy wound was the application of a plastic silo (the 'Bogota bag'). This system is inexpensive, readily available and preserves the intact fascia when sutured to the skin edges. However, because the plastic silo does not provide sufficient traction to the wound edges and allows the fascial edges to retract laterally, the abdominal cavity loses part of its volume or domain resulting in difficult fascial closure under significant tension, especially if the closure is delayed beyond the first week. 
In 1995, the vacuum pack method utilizing a polyethylene sheet tucked between the parietal peritoneum and the bowel was introduced. The improvement with this technique compared to earlier methods was related to the prevention of the formation of adhesions between the abdominal wall and the bowel [6]. A further improvement described in 2001 was the introduction of the vacuumassisted wound management concept [7]. The application of vacuum-assisted wound closure techniques to open abdomens helps nursing care and is associated with the highest rate of subsequent delayed primary fascial closure and lowest mortality [8]. Even in the management of the most severe complication of the open abdomen, the exposed enteric fistula, vacuum-assisted wound management is able to control fistula secretion allowing the wound around it to heal [9]. A variety of 'self-made' topical negative pressure dressings utilizing the same principle has been described [10].

In some institutions, absorbable mesh is used for temporary cover of laparostomies, but the risks of prosthesis infection and fistula formation are still considerable. In a single institution, prospective randomized study comparing polyglactin 910 mesh and vacuum-assisted closure in 51 patients with laparostomy [11], the fistula rate was $21 \%$ after vacuum-assisted closure and 5\% after mesh (statistically not significant). There were no differences in mortality, intra-abdominal infection, or delayed primary fascial closure rates (26\% and 31\%). The authors found both methods to be useful and equally likely to produce delayed fascial closure [11].

The likelihood of fascial closure is also related to the underlying etiology. In a study of 71 patients requiring laparostomy for gastrointestinal sepsis, pancreatitis or trauma, only $20 \%$ achieved definitive fascial closure [12]. The likelihood of fascial closure was significantly higher in trauma patients.

A recent modification combines the use of a mesh and vacuum-assisted closure by using a temporary mesh sutured to the fascial edges under the vacuum with gradual tightening of the mesh at dressing changes until the fascia can be closed primarily [13]. Currently, this technique is the preferred method of temporary abdominal closure at our institution (Figure 1).

\section{Classification of open abdomen}

Because of the multitude of conditions leading to open abdomen, the comparison of different series and treatment outcomes has been difficult. Recently, a consensus group established a new classification system for open abdomen [14]. The criteria for different categories are based on the degree of contamination and adherence between bowel and abdominal wall or 'fixity' (lateralization of the abdominal wall). Among the four categories, Grade 1 refers to clean (1A) or contaminated (1B) wound without adherence, and $2 \mathrm{~A}$ and $2 \mathrm{~B}$ to clean and contaminated wounds with adherence, respectively. Grade 3 is an open abdomen complicated by fistula formation, and grade 4 a frozen abdomen.

\section{Definitive abdominal wall closure}

The primary aim in managing laparostomy patients is to achieve primary fascial closure as soon as possible without causing recurrent abdominal compartment syndrome or other complications associated with premature closure. If the infection source has been controlled and even if a relaparotomy might be needed in the near future, every effort should be made to achieve primary fascial closure during the initial hospitalization period and avoid the significant morbidity associated with leaving the abdomen open for delayed reconstruction. Gradual fascial closure, often mesh-assisted, seems currently to be the best available technique, but other possibilities, such as the components separation technique at an early stage [15], or fascial closure with a mesh prosthesis can be considered when there is no infection and enough skin to cover the prosthesis. However, if primary fascial closure is not possible, an early decision to resort to the planned hernia strategy is a good option.

A planned hernia approach aims at skin coverage with subsequent delayed abdominal wall reconstruction. The skin closure is most often achieved with autologous splitthickness skin grafting over the exposed bowel. Conditions favoring a planned hernia strategy include the inability to re-approximate the retracted abdominal wall edges, sizeable tissue loss, risk of tertiary abdominal compartment syndrome, inadequate infection source control, anterior enteric fistula, and poor nutritional status of the patient. The maturation of the skin graft requires about 9-12 months, after which the grafted skin can be easily removed from the bowel surface without additional iatrogenic lesions. Large abdominal wall defects can be reconstructed with pedicular or microvascular flaps. The most commonly used is the tensor fascia lata (TFL)-flap [16].

\section{Does laparostomy improve outcome?}

The potential benefits of laparostomy have been most extensively studied in patients with secondary peritonitis. In a small randomized study of 40 patients comparing open treatment utilizing a polypropylene mesh for temporary cover with closed treatment, there was no significant difference in postoperative acute renal failure, duration of mechanical ventilatory support, need for total parenteral nutrition, rate of residual infection, or need for reoperation for residual infection [17]. Even though the difference in mortality (55\% vs 30\% favoring closed treatment) was not statistically significant, the study was terminated at the first interim analysis due to 

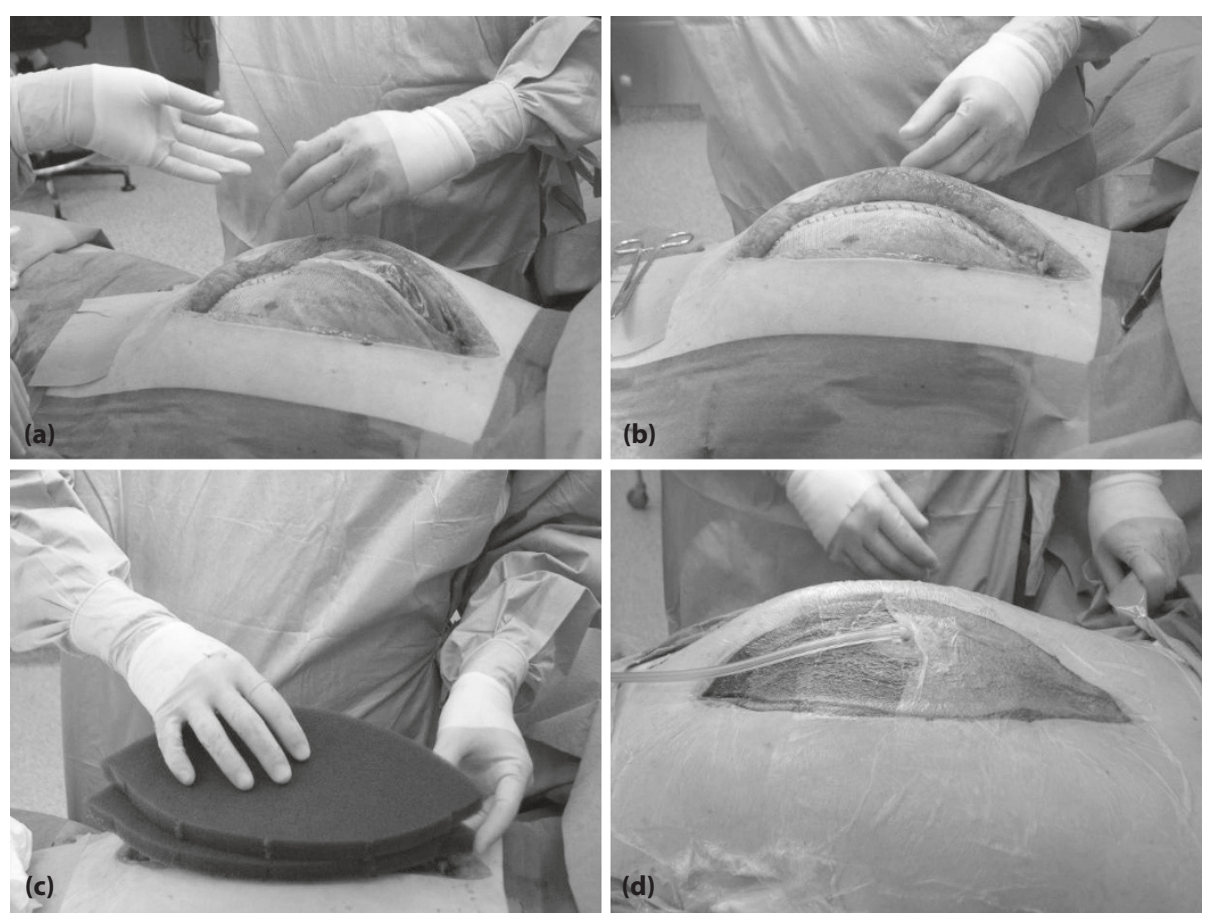

Figure 1. (a-d) Mesh-assisted vacuum-assisted closure dressing.

the clear tendency (relative risk and odds ratio for death 1.83 and 2.85 higher in the open group) toward a more favorable outcome after closed treatment. The authors concluded that closed management of the abdomen may be a more rational approach.

The benefits of laparostomy in intra-abdominal sepsis are conceptually related to the policy toward relaparotomies; should a relaparotomy be performed as a planned second look decided on already at the initial operation, or should relaparotomy only be performed ondemand after identifying a surgical complication (abscess, suture line or anastomotic leak) not amenable to percutaneous drainage. A recent, well-conducted randomized study comparing an on-demand to a planned relaparotomy strategy in patients with severe peritonitis showed that the on-demand group had a substantial reduction in relaparotomies, health care utilization, and medical costs [18]. There were, however, no significant differences in mortality or major peritonitis-related morbidity.

The current consensus does not support laparostomy and planned relaparotomy as the routine strategy in secondary peritonitis [19]. There are, however, some patient groups where laparostomy is unavoidable or practical. As has been lineated by Moshe Schein, one of the true pioneers in open abdomen, there are abdomens that cannot be closed due to major abdominal wall tissue loss, poor condition of the fascia, or extreme visceral or retroperitoneal swelling, and there are abdomens that should not be closed either to avoid abdominal compartment syndrome or because of a planned reoperation within a day or two (why lock the gate through which you are to re-enter very soon?) [20].

Infected pancreatic necrosis is an established indication for surgical necrosectomy in patients with severe acute pancreatitis. Although minimally invasive necrosectomy is feasible in some patients, the golden standard is still open necrosectomy $[21,22]$. While open necrosectomy is performed in a more or less identical fashion, there are four techniques, differing in the way they provide exit channels for further slough and infected debris: Open packing, planned relaparotomies, closed packing, and closed continuous lavage [22]. Although mortality rates below $15 \%$ have been reported after all four techniques, necrosectomy and subsequent closed continuous lavage of the lesser sac seems to be associated with the lowest morbidity [22].

The benefits of laparostomy in the management of abdominal compartment syndrome in patients with severe acute pancreatitis have not been reliably demonstrated. Although there is no question that opening the abdomen reduces intra-abdominal pressure (IAP) in this patient group, the indications for, techniques used, subsequent management of the open abdomen, and potential risk of increased infectious complications are highly controversial. In a collective review of 250 patients undergoing midline laparostomy, decompression had an 
overall positive effect on hemodynamic, respiratory, and renal functions [23]. Central venous pressure (CVP) and pulmonary artery pressure decreased, most likely caused by the direct effect of the decrease in IAP on the thoracic cavity. Cardiac function improved in the majority of the patients. There was an improvement in $\mathrm{PaO}_{2} / \mathrm{FiO}_{2}$ ratio and a decrease in peak airway pressure, but the respiratory function remained severely impaired in most patients. Significant improvement in urinary output was observed in all but two studies.

In a report from our institution, among the 26 patients with severe acute pancreatitis undergoing surgical decompression for abdominal compartment syndrome during the past 6 years, mostly using a full-thickness midline laparostomy, the median sequential organ failure assessment (SOFA) score at the time of decompression was 12 , interquartile range (IQR) $10-15$, and the median IAP was 31.5 (IQR 27-35) mmHg [24]. After decompression, 14 (54\%) patients had improved renal or respiratory functions. The overall mortality rate was $46 \%$, but in 17 patients in whom decompression was performed within the first 4 days from disease onset, the mortality rate was $18 \%$. We concluded that in patients with severe acute pancreatitis and abdominal compartment syndrome, surgical decompression may improve renal or respiratory functions, and when performed early surgical decompression is associated with reduced mortality [24].

Leaving the abdomen open after a damage control procedure for trauma is an essential component of the abbreviated laparotomy and planned reoperation strategy. Although there are no randomized studies showing that the damage control approach improves outcome in abdominal trauma patients with severely deranged physiology, cumulative material from 1001 damage control patients demonstrated a $50 \%$ mortality rate [25]. This seems high, but a $50 \%$ survival rate in this very sick patient population is remarkable. More recent studies have shown other benefits of damage control in trauma patients. In a series of patients with severe abdominal injuries compared with historical controls from Atlanta, damage control use increased from $7 \%$ to $18 \%$ and the overall mortality decreased from $76 \%$ to $27 \%$ [26]. A similar decrease was noted in another study from Philadelphia where the mortality rate after the paradigm change decreased from $42 \%$ to $10 \%$ [27].

Survival after damage control, however, comes with a price. In a series of 334 damage control patients, 276 of whom survived to abdominal closure, there was a $25 \%$ incidence of wound infections, abscesses, and enteric fistulas [28]. In the two studies mentioned previously, the incidence of abscesses was $14 \%$ and $18 \%$, and of fistulas $18 \%$ and $14 \%$, respectively $[26,27]$. In a series of 56 trauma patients with early mortality of $27 \%, 31$ patients required subsequent treatment for complications related to the open abdomen; overall, 58 late operations for complications were performed, most commonly for infection (46\%), hernia (41\%) and enteric fistula (34\%) [29].

\section{Conclusion}

Open abdomen is a situation that is encountered increasingly frequently in trauma and emergency surgery, and is often the price to be paid for saving severely ill or injured patients. Current evidence supports the use of laparostomy in all patient groups with severe abdominal compartment syndrome. Obviously, the inability to close the abdomen due to tissue loss or extreme swelling is a mandatory indication for laparostomy. Open abdomen treatment of patients with secondary peritonitis or infected pancreatic necrosis to facilitate the clearing of the infection seems unwarranted. A relative indication for laparostomy is the planned return to the operating room for relaparotomy within 1-2 days where closing the wound at the initial operation requires more time and poses an additional risk to the integrity of the fascia. With modern techniques of temporary abdominal closure, the risks of enteric fistulas or failure to close the fascia are acceptable.

\section{Abbreviations}

$\mathrm{CVP}=$ central venous pressure, $\mathrm{IAH}=$ intra-abdominal hypertension, $\mathrm{IAP}=$ intra-abdominal pressure, IQR = interquartile range, SOFA = sequential organ failure assessment, TFL-flap = tensor fascia lata flap.

\section{Competing interests}

The author declares that they have no competing interests.

\section{Published: 9 March 2010}

\section{References}

1. Steinberg D: On leaving the peritoneal cavity open in acute generalized suppurative peritonitis. Am J Surg 1979, 137:216-220.

2. Schein M, Saadia R, Decker GGA: The open management of septic abdomen. Surg Gynecol Obstet 1986, 163:587-592.

3. Bradley El III: Management of infected pancreatic necrosis by open drainage. Ann Surg 1987, 206:542-548.

4. Rotondo MF, Schwab CW, McGonigal MD, et al:. "Damage control." An approach for improved survival in exsanguinating penetrating abdominal injury. J Trauma 1993, 35:375-83.

5. Cheatham ML, Malbrain MLNG, Kirkpatrick A, et al:: Results from the international conference of experts on intra-abdominal hypertension and abdominal compartment syndrome. II. Recommendations. Intensive Care Med 2007, 33:951-62.

6. Brock WB, Barker DE, Burns RP: Temporary closure of open abdominal wounds: the vacuum pack. Am Surg 1995, 61:30-35.

7. Garner GB, Ware DN, Cocanour CS, et al:. Vacuum-assisted wound closure provides early fascial reapproximation in trauma patients with open abdomens. Am J Surg 2001, 182:630-638.

8. van Hensbroek PB, Wind J, Dijkgraaf MGW, Busch ORC, Goslings JC: Temporary closure of the open abdomen: A systematic review on delayed primary fascial closure in patients with open abdomen. World J Surg 2009, 33:199-207.

9. Becker HP, Willms A, Schwab R: Small bowel fistulas and the open abdomen. Scand J Surg 2007, 96:263-271.

10. Leppäniemi A: Open abdomen after severe acute pancreatitis. Eur J Trauma Emerg Surg 2008, 34:17-23.

11. Bee TK, Croce MA, Magnotti LJ, et al.: Temporary abdominal closure 
techniques: A prospective randomized trial comparing polyglactin 910 mesh and vacuum-assisted closure. J Trauma 2008 65:337-344.

12. Tsuei BJ, Skinner JC, Bernard AC, Kearney PA, Boulanger BR: The open peritoneal cavity: Etiology correlates with the likelihood of fascial closure. Am Surg 2004, 70:652-656.

13. Petersson U, Acosta S, Björck M: Vacuum-assisted wound closure and meshmediated fascial traction - a novel technique for late closure of the open abdomen. World J Surg 2007, 31:2133-2137.

14. Björck M, Bruhin A, Cheatham M, et al: Classification - Important step to improve management of patients with an open abdomen. World J Surg 2009, 33:1154-1157.

15. Ramirez OM, Ruas E, Dellon AL: Components separation method for closure of abdominal-wall defects: and anatomic and clinical study. Plast Reconstr Surg 1990, 86:519-526.

16. Lyle WG, Gibbs M, Howdieshell TR: The tensor fascia lata free flap in staged abdominal wall reconstruction after traumatic evisceration. J Trauma 1999, 46:519-522.

17. Robledo FA, Luque-de-Leon E, Suarez R, et al:: Open versus closed management of the abdomen in the surgical treatment of severe secondary peritonitis: a randomized clinical trial. Surg Infect 2007, 8:63-71.

18. van Ruler O, Mahler CW, Boer KR, et al.: Comparison of on-demand vs planned relaparotomy strategy in patients with severe peritonitis. JAMA 2007, 298:865-873.

19. Pieracci FM, Barie PS: Management of severe sepsis of abdominal origin. Scand J Surg 2007, 96:184-196.

20. Schein $M$ : Surgical management of intra-abdominal infection: is there any evidence? Langenbecks Arch Surg 2002, 387:1-7.

21. Connor S, Raraty MGT, Howes N, et al:: Surgery in the treatment of acute pancreatitis - minimal access pancreatic necrosectomy. Scand J Surg 2005, 94:135-142.
22. Werner J, Hartwig W, Hackert T, Buchler MW: Surgery in the treatment of acute pancreatitis - open pancreatic necrosectomy. Scand J Surg 2005, 94:130-134

23. De Waele JJ, Hoste EA, Malbrain ML: Decompressive laparotomy for abdominal compartment syndrome - a critical analysis. Crit Care 2006, 10:R51.

24. Mentula P, Hienonen P, Kemppainen E, Puolakkainen P, Leppäniemi $A$ : Surgical decompression for abdominal compartment syndrome in severe acute pancreatitis. Arch Surg 2010, (in press).

25. Shapiro MB, Jenkins DH, Schwab CW, Rotondo MF: Damage control: collective review. J Trauma 2000, 49:969-978.

26. Nicholas JM, Parker Rix E, Easley KA, et al.: Changing patterns in the management of penetrating abdominal trauma: The more things change, the more they stay the same. J Trauma 2003, 55:1095-1110.

27. Johnson JW, Gracias VH, Schwab CW, et al.: Evolution in damage control for exsanguinating penetrating abdominal injury. J Trauma 2001, 51:261-271.

28. Miller RS, Morris JA Jr, Diaz JJ Jr, Herring MB, May AK: Complications after 344 damage-control open celiotomies. J Trauma 2005, 59:1365-1374.

29. Sutton E, Bochicchio GV, Bochicchio K, et al:. Long term impact of damage control surgery: a preliminary prospective study. J Trauma 2006 61:831-836

doi:10.1186/cc8857

Cite this article as: Leppäniemi AK: Laparostomy: why and when? Critical Care 2010, 14:216 